\title{
Upregulation of actin-like gene expression in giant freshwater prawns Macrobrachium rosenbergii infected with Metschnikowia bicuspidata*
}

\author{
Hung-Yi Su${ }^{1}$, Pei-Chi Wang ${ }^{2}$, Yi-Yang Lien ${ }^{2}$, Ming-An Tsai ${ }^{2}$, Shyh-Shyan Liu ${ }^{2}$, \\ Kun-Chan Wu' ${ }^{1}$, Cheng-Hung Hsieh ${ }^{1}$, Shih-Chu Chen ${ }^{2, * *}$ \\ ${ }^{1}$ Institute of Biotechnology, and ${ }^{2}$ Department of Veterinary Medicine, National Pingtung University of Science and \\ Technology, Pingtung 91201, Taiwan, ROC
}

\begin{abstract}
The giant freshwater prawn Macrobrachium rosenbergii is commercially cultured throughout the world including Taiwan. From 1992 to 1995, Taiwanese production decreased by approximately $50 \%$ due to disease. The yeast Metschnikowia bicuspidata is considered to be one of the major causes of white muscle disease, but the molecular mechanism of its pathogenesis is not known. Using RNA differential display (DD) with muscle and hepatopancreatic tissue, we identified a 324 nucleotide (nt) message specifically expressed by $M$. rosenbergii infected with $M$. bicuspidata but not in the controls. A ribonuclease protection assay (RPA) confirmed expression in both tissues. RPA data also revealed an additional $230 \mathrm{bp}$ mRNA message that was not identified by DD. Using RNA ligase-mediated rapid amplification of $5^{\prime}$ cDNA ends (5'-RACE), we successfully isolated a 1357 bp full-length gene (c57) that showed 92 and $87 \%$ sequence identity to the actin gene of the Kuruma shrimp Marsupenaeus japonicus (also called Penaeus japonicus) (GenBank accession number AB055975) and the beta-actin gene of the white shrimp Litopenaeus vannamei (also called Penaeus vannamei) (GenBank accession number AF300705), respectively. The deduced amino acid sequence of $c 57$ showed $83 \%$ sequence similarity to $M$. japonicus and L. vannamei actin proteins. Based on this high homology, we suggest that upregulation of actin expression in the muscle and hepatopancreas is part of the shrimp response to $M$. bicuspidata infection. Increased expression may be related to repair of tissues damaged by yeast infection.
\end{abstract}

KEY WORDS: Macrobrachium rosenbergii $\cdot$ Metschnikowia bicuspidata $\cdot$ Actin Resale or republication not permitted without written consent of the publisher

\section{INTRODUCTION}

Commercial culture of the giant freshwater prawn Macrobrachium rosenbergii is undertaken throughout the world including Taiwan, where production decreased by 47 to $52 \%$ from $1992-1995$ due to diseases (New 1995) caused by yeast infection and Lactococcus

\footnotetext{
*The nucleotide sequence data reported in this paper has been submitted to GenBank and has been assigned accession number AY651918
}

infection (Cheng \& Chen 1998, Lu et al. 1998). Yeast infections are associated with gross signs including yellow exoskeletons, a swollen hepatopancreas, milky hemolymph and opaque, whitish muscles (Cheng \& Chen 1998, Lu et al. 1998). Yeast disease outbreaks have occurred since 1988 in southern Taiwan, most frequently in the cool season (i.e. October to March) and predominantly (i.e. $64 \%$ ) during the winter (i.e. December to February) since 1994 (Lu et al. 1998). The rate of infection is the highest in adult prawns (i.e. $73 \%)$ and lower in juveniles $(25 \%)$ and postlarvae 
$(2 \%)$, while no infections are found in larvae (Lu et al. 1998).

Yeasts reported to be pathogenic for Macrobrachium rosenbergii include Candida sake I and II, Pichia anoma, Endomyces fibuliger and Candida famata (Lu et al. 1998). As identification of pathogenic yeast species based on cellular morphology and biochemical tests are often strain-specific (Lu et al. 1998), molecular approaches such as those based on the 26S rDNA gene (Kurtzman \& Robnett 1997) are more useful. We used both approaches to identify individual species from coinfections of Enterococcus faecium and Metschnikowia bicuspidata in M. rosenbergii (Chen et al. 2003). However, the host response to $M$. bicuspidata infection and the mechanism of yeast pathogenicity have not been studied.

The technique of ribonucleic acid (RNA) differential display (DD) (Liang \& Pardee 1992) was first used to study differences in gene expression during various developmental stages of tumor cells. In the present study, we use the technique to identify a gene that is upregulated in muscle and hepatopancreatic tissues of Macrobrachium rosenbergii infected with Metschnikowia bicuspidata.

\section{MATERIALS AND METHODS}

Diseased prawn samples. Diseased prawn samples (body weight 5 to $8 \mathrm{~g}$, body length 8 to $10 \mathrm{~cm}$ ) were collected for molecular analyses from 4 prawn farms (10 prawns were randomly selected from each farm) during disease outbreaks in 2001 (Chen et al. 2003). Infection with Metschnikowia bicuspidata was confirmed by sequence analysis of the D1/D2 domain of $26 \mathrm{~S}$ rDNA and histopathological examination in diseased prawn tissues (Chen et al. 2003).

Yeast strains. The purified isolate derived from infected prawns in this study was as described by Chen et al. (2003) and labeled Metschnikowia biscupidata AOD081MB.

Experimental infections. Healthy Macrobrachium rosenbergii (body weight 5 to $7 \mathrm{~g}$ ) obtained from 1 prawn farm in Pingtung, Taiwan, were held at a density of 120 prawns in 3 continuously aerated 4001 aquaria containing $300 \mathrm{l}$ of fresh water at $25^{\circ} \mathrm{C}$ for $7 \mathrm{~d}$, until the prawns were acclimated to laboratory conditions. These prawns were fed twice daily with a commercial diet (Prawn Diet, Gold Brand Feed) and the waste was removed daily. Subsequently, 20 prawns with similar body length ranges were evenly divided into 2 groups: the Treatment Group and the Control Group. Ten-fold serial dilutions of the purified pathogen, isolate AOD081MB (Chen et al. 2003) (optical density $[\mathrm{OD}]=1$ at $620 \mathrm{~nm}$ ), were prepared in sterile normal saline $(0.85 \% \mathrm{NaCl})$. Each prawn in the Treatment Group was injected intramuscularly (IM) with $0.1 \mathrm{ml}$ of the diluted pathogen (approximately $1 \times 10^{7}$ AOD081MB), whilst the Control Group was injected IM with $0.1 \mathrm{ml}$ of sterile normal saline. After injection, the groups were maintained separately in a 801 aquaria under the same conditions as described above for acclimation.

RNA isolation and mRNA differential display. In this study, the technique of mRNA DD was used to investigate changes in gene expression in muscle and hepatopancreatic tissue $6 \mathrm{~h}$ after IM injection in the Treatment and Control Groups. Total RNA was isolated from muscle and hepatopancreatic tissue samples derived from injection control, injection challenge and naturally infected prawns (10 prawns in each group) using 3-Zol reagent (MDBio). Pooled RNA from muscle or hepatopancreatic tissue from each group was used for DD analysis. This RNA was treated with DNase I and quantified spectrophotometrically by absorbance at $260 \mathrm{~nm}$. RNA integrity was verified by $1 \%$ agarose gel electrophoresis followed by ethidium bromide staining (MDBio). Synthesis of the first strand cDNA was accomplished by reverse transcription (RT) using $300 \mathrm{U}$ of Moloney murine leukemia virus reverse transcriptase (MMLV-RT) (Life Technologies) and $2.5 \mu \mathrm{M}$ of 3 '-primer. After heat inactivation of the RT at $95^{\circ} \mathrm{C}$ for $5 \mathrm{~min}, 2 \mu \mathrm{l}$ of the sample was added to $18 \mu \mathrm{l}$ of polymerase chain reaction (PCR) mix containing a 5'-primer and AmpliTaq DNA polymerase (Perkin Elmer). The PCR mix preparation was based on a nonradioactive procedure described by Doss (1996) except that the amount of AmpliTaq DNA polymerase was doubled. As DNase I-treated RNAs are sometimes contaminated with residual chromosomal DNA (Liang et al. 1993), a control RT-PCR without reverse transcriptase was included. The RT-PCR products from each sample were run on a $5 \%$ denaturing polyacrylamide gel stained with silver (Su et al. 2004). Four $3^{\prime}$-primers (5'- T $\left._{12} \mathrm{AT}-3^{\prime}, \quad 5^{\prime}-\mathrm{T}_{12} \mathrm{CA}-3^{\prime}, \quad 5^{\prime}-\mathrm{T}_{12} \mathrm{CC}-3^{\prime}, \quad 5^{\prime}-\mathrm{T}_{12} \mathrm{CG}-3^{\prime}\right)$ and four 5'-primers (5'-AGTAGCTGGA-3', 5'-CTCGGTACAC-3', 5'-ATGTGGTGGT-3', 5'-TCTGCCGTGA-3') (Su et al. 2004) synthesized by MDBio were used to accomplish 16 RT-PCRs. Differentially expressed gene product sizes were determined based on the procedures described by Bassam et al. (1991) using silver-stained gels with pGEM DNA markers obtained from Promega. DNA fragments that were differentially present in the sample groups were recovered from the gel using the methods of Weaver et al. (1994). The recovered DNA was PCR-reamplified and cloned using pCRII vector (Invitrogen) in Escherichia coli INV $\alpha$ F competent cells (Invitrogen) and the transformed cells were recovered in SOC medium (Invitrogen). Plasmid DNA was purified from selected trans- 
formants by a modified mini alkaline-lysis and polyethylene glycol (PEG) precipitation procedure (PerkinElmer) using Terrific Broth (Tartof \& Hobbs 1987) for the growth of bacteria and including $\mathrm{PEG}_{8000}$ in the precipitation step. Six independent clones from each original DNA fragment were sequenced with M13 forward and reverse primers using an automated sequencer (MDBio). The complete nucleotide sequence data of differentially expressed cDNA inserts were determined by aligning both strands of sequence data using the DNAMAN Program (Version 2.5, Lynnon BioSoft). Sequences were tested for homology to known sequences at GenBank using the basic local alignment search tool (BLAST) (Altschul et al. 1990).

Amplification of 5' cDNA ends. Total RNAs were extracted from muscle and hepatopancreatic tissue of experimentally inoculated and naturally infected prawns using Trizol reagent (Life Technologies). Isolation of the full-length differentially expressed cDNA clone was accomplished by 5'-RACE using a GeneRacer Kit (Version J, Invitrogen) according to the manufacturer's instructions, except that the sequences of a 3' reverse gene-specific primer (5'-GTG GGG CAA TGA TCT TGA TGT GGG GAG TAG GAC TGG TAA CTG CAG-3') were designed from the differentially expressed cDNA sequence. After performing 5-RACE, the PCR product was cloned in pCRII vector (Invitrogen). Plasmids from positive colonies were purified and sequenced by MDBio. The resulting sequences were tested for homology to known sequences at GenBank using BLAST (Altschul et al. 1990).

Ribonuclease protection assay. A non-radioactive ribonuclease protection assay (RPA) was performed to confirm the expression of $c 57$ cDNA in the muscle and hepatopancreatic tissue of infected prawns using an RPA III kit (Ambion) following the manufacturer's recommended protocol and the method described by Wundrack \& Dooley (1992). The antisense RNA probe was prepared by transcription of an ApaI-linearized plasmid template of $c 57$ with Sp6 RNA polymerase using the Sp6/T7 transcription kit (Boehringer Mannheim) in the presence of digoxigenin (DIG)-UTP (Roche Molecular Biochemicals). The 464 nucleotide (nt) full-length hybridization probe contained 324 nt of 3' coding sequence of cloned c57 RNA and 140 nt of vector sequence. This antisense riboprobe was subsequently purified using a $1 \mathrm{ml}$ Sephadex G-50 (Sigma) spin column as previously described ( $\mathrm{Su}$ et al. 2001). After hybridization with RNA from muscle and hepatopancreatic tissue, the unhybridized single-stranded RNA was digested by RNases A and T1 (provided with the RPA III kit). Protected fragments were separated on a $6 \%$ polyacrylamide gel containing urea, transferred to a nylon membrane by electroblotting, and detected using a DIG Nucleic Acid Detection kit (Roche Molecular Biochemicals).

\section{RESULTS AND DISCUSSION}

\author{
Identification and isolation of $c 57$ from \\ diseased prawns
}

Using the primers, $5^{\prime}-\mathrm{T}_{12} \mathrm{AT}-3^{\prime}$ and $5^{\prime}$-ATGTGGTGGT-3', 1 up-regulated gene (c57) was identified in muscle and hepatopancreatic tissue of experimentally (Fig. 1, lanes 2 and 3 and 4 and 5, respectively) and naturally infected prawns (Fig. 1, lanes 8 and 9 and lane 10, respectively), but not in the tissues of healthy prawns (Fig. 1, lanes 6 and 7, respectively). As repeated experiments showed a similar display pattern (data not shown), c57 was selected for further work. Using the other 15 primers, 3 additional fragments were found in Metschnikowia bicuspidata-infected prawns but not consistently in repeated experiments

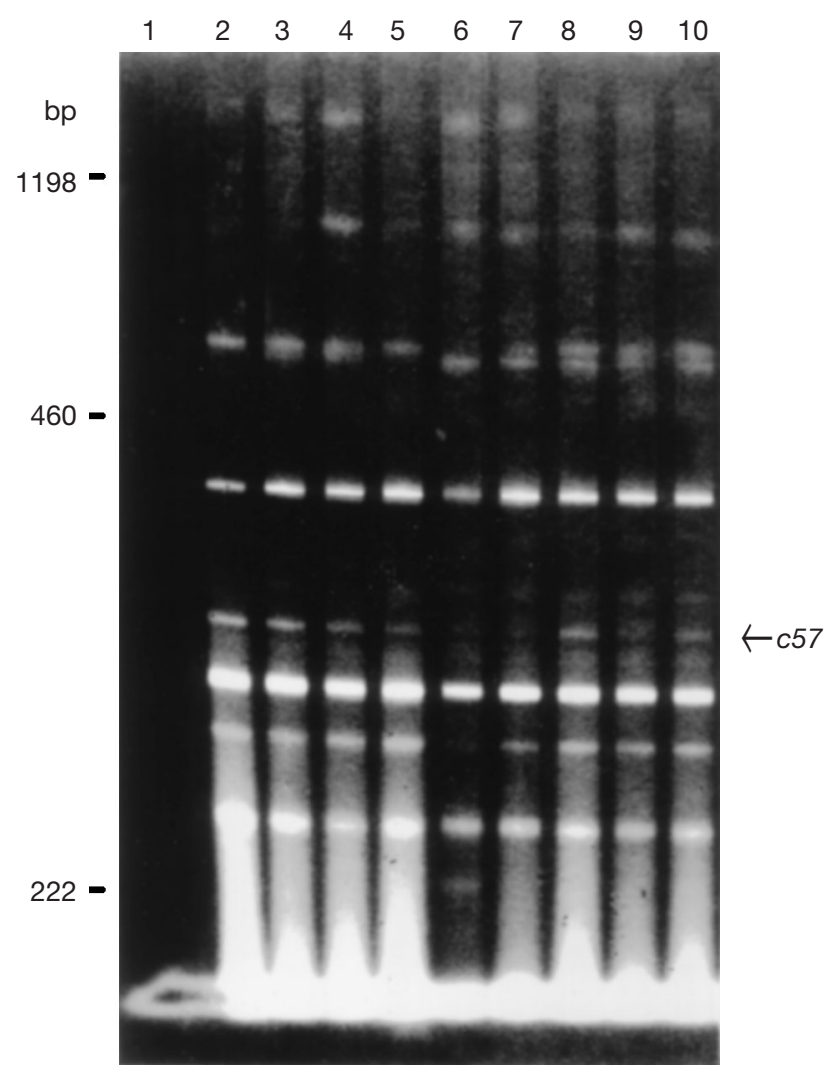

Fig. 1. Identification and isolation of c57 from muscle and hepatopancreas of Metschnikowia bicuspidata-infected Macrobrachium rosenbergii prawns by differential display (DD). Reverse transcriptase-polymerase chain reaction (RT-PCR) results of total RNAs isolated from hepatopancreatic (lanes 2 and 3) and muscle (lanes 4 and 5) tissues of experimentally injected prawns, hepatopancreas (lanes 8 and 9) and muscle (lane 10) of naturally infected prawns, and hepatopancreas (lane 6) and muscle (lane 7) of healthy prawns. Lane 1: results of RT-PCR without reverse transcriptase with template comrising pooled hepatopancreatic RNA from experimentally infected prawns 
(data not shown). Thus, they were excluded from further investigation. RNA sample authenticity was confirmed by the absence of cDNA amplification using pooled hepatopancreatic RNA from experimentally infected prawns in the absence of reverse transcription (Fig. 1, lane 1). Thus, cDNA products were fingerprints of tissue-derived mRNAs.

\section{Nucleotide and amino-acid sequences of $c 57$}

The c57 fragment from a display gel was eluted, PCR-reamplified, cloned and sequenced from both strands. Sequence alignment revealed $100 \%$ complementarity between the 2 strands (data not shown). The sequence (nt 1027 to 1348) is shown in Fig. 2A with the flanking primer set shown in bold italics. There is 1 nucleotide mismatch at the penultimate base (lowercase $g$, Fig. 2A). This mismatch between the primer and $c 57$ suggested that the primer may exhibit degeneracy during annealing in the RT step (Liang et al. 1993).

The full-length c57 sequence (Fig. 2A) obtained by 5 '-RACE had $92 \%$ identity to the nucleotide sequence of the actin gene of the Kuruma shrimp Marsupenaeus japonicus (also called Penaeus japonicus) (GenBank accession number $\mathrm{AB}$ 055975) and $87 \%$ identity to the beta-actin gene of white shrimp Litopenaeus vannamei (also called Penaeus vannamei) (GenBank accession number AF300705). The deduced amino acid sequence of $c 57$ shared $83 \%$ sequence similarity with residues of 78-381 of the $M$. japonicus and $L$. vannamei actin proteins (Fig. 2B).

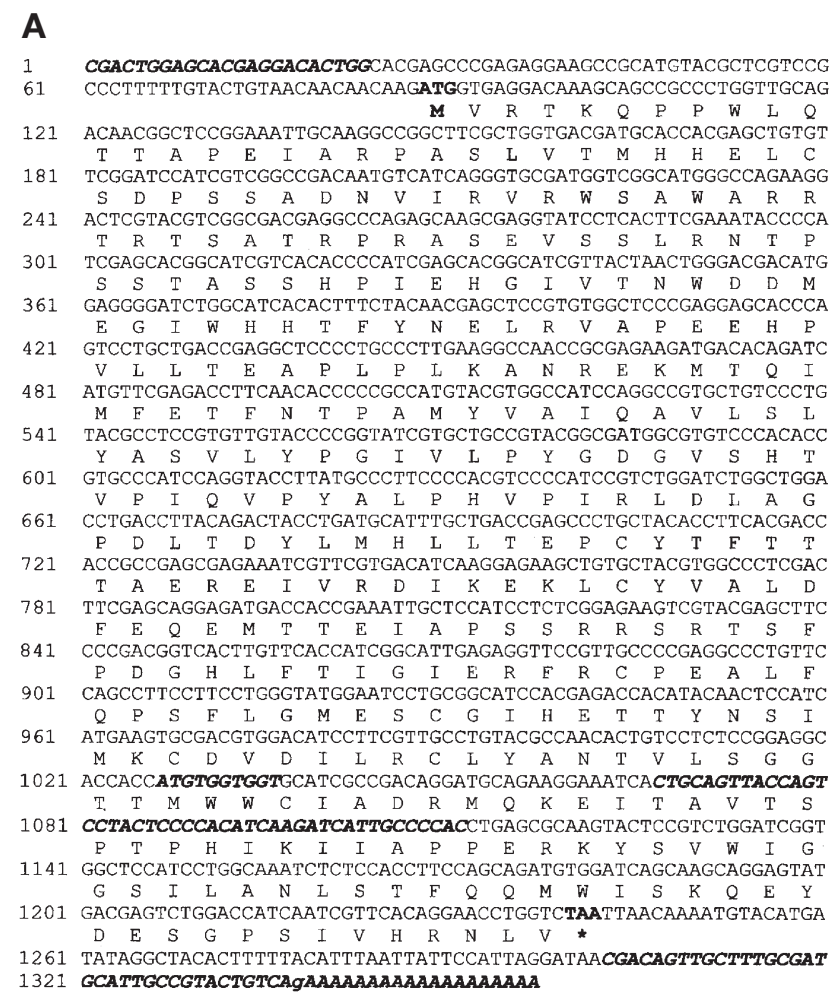

B

C57. PRO MVRTKOPPWLQTTAPEIARPASLVTMHHELCSDPSSADNVIRVRWSAWARRTRTSATRPRASEVSSLRNTPSSTASS-----HPIEHGIVTNWDDMEGIWHHTEYNELRV 105 MC-- DDEVALLVDNGSGMCKA.GFAGDDAPRAVFPSIVGRPHQGVMVGGQKDSYVGDEAQSKRGILTLKYPIEHGIVINWDDMEKIWHHTEYNELRV 97

C57.PRO APEEHPVLLTEAPLPLKANREKMTQIMFETENT PAMYVAIQAVLSLYASVLYPGIVLPYGDGVSHTVPIQVPYALPHVPIRLDLAGPDLTDYLMHLITEPCYTFTTTAER 215 M. japonicus. PRO APEEHPVLLTEAPLNPKANREKMTQIMFETENTPAMYVAIQAVLSLYASGRTTGIVLDSGDGVSHTVPIYEGYALPHAILRLDLAGRDLTDYLMKILTERGYTETTTAER 207 P. Vannamei.PRO APEEHPVLLTEAPLNPKANREKMTQIMFETENTPAMYVAIQAVLSLYASGRTTGIVLDSGDGVSHTVPIYEGYALPHAILRLDLAGRDLTDYLMKILTERGYTFTTTAER 207

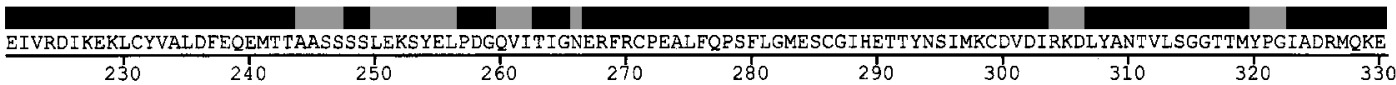

657. PRO EIVRDIKEKLCYVALDFEQEMTTEIAPSSRRSRTSFPDGHLFTIGIERERCPEALFQPSFLGMESCGIHETTYNSIMKCDVDILRCLYANTVLSGGTTMWWCIADRMOKE 325 M.japonicus. PRO EIVRDIKEKLCYVALDFEQEMTTAASSSSLEKSYELPDGQVITIGNERERCPEALFQPSFLGMESCGIHETTYNSIMKCDVDIRKDLYANTVLSGGTTMYPGIADRMQKE 317 P. Vannamei.PRO EIVRDIKEKLCYVALDEEQEMT TAASSSSLEKSYELPDGQVITIGNERFRCPEALFQPSFLGMESCGIHEITYNSIMKCDVDIRKDLYANTVLSGGTTMYPGIADRMQKE 317

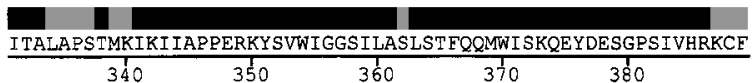

657. PRO ITAVTSPTPHIKIIAPPERKYSVWIGGSILANLSTFQOMWISKQEYDESGPSIVHRNLV M. Japonicus. PRO ITALAPSTMKIKIIAPPERKYSVWIGGSILASLSTEQQMWISKQEYDESGPSIVHRKCF P. Vannamei.PRO IPALAPSTMKIKIIAPPERKYSVWIGGSILASLSTEQQMWISKQEYDESGPSIVHRKCF

Fig. 2. Nucleotide sequence (GenBank accession number AY651918) and deduced amino acid sequence of c57 from Macrobrachium rosenbergii infected with Metschnikowia bicuspidata. (A) Start and stop codons are shown in bold letters. Nucleotide sequences of flanking mRNA mapping primers are shown in bold italics. Nucleotides 320 to 1070 showed $92 \%$ identity to the Kuruma shrimp Marsupenaeus japonicus actin gene and $87 \%$ identity to the white shrimp Litopenaeus vannamei beta-actin gene. *: stop codon. (B) Data from alignment of the deduced protein sequence of $c 57$ showing $83 \%$ similarity (highlighted regions) to the M. japonicus actin and Penaeus vannamei beta-actin protein sequences. PRO: protein sequence to each corresponding DNA 


\section{Confirmation of $c 57 \mathrm{mRNA}$ expression in the hepatopancreas and muscle}

Using nonradioactive RPA to verify c57 mRNA expression in the hepatopancreas and muscle of infected prawns (Fig. 3), it was found that hybridization of a DIG-labeled c57 antisense RNA probe to RNA from infected hepatopancreatic and muscle tissue resulted in the formation of a $324 \mathrm{bp}$ RNA-RNA hybrid that was protected from subsequent RNase digestion (Fig. 3, lanes 2 to 5). Expression of $c 57$ detected by RPA was similar to that for the DD (Fig. 1). Visible changes such as whitish muscles and massive lesions have been found in Macrobrachium rosenbergii after Metschnikowia bicuspidata infection (Lu et al. 1997, 1998). It is possible that $c 57$ was upregulated in response to yeast

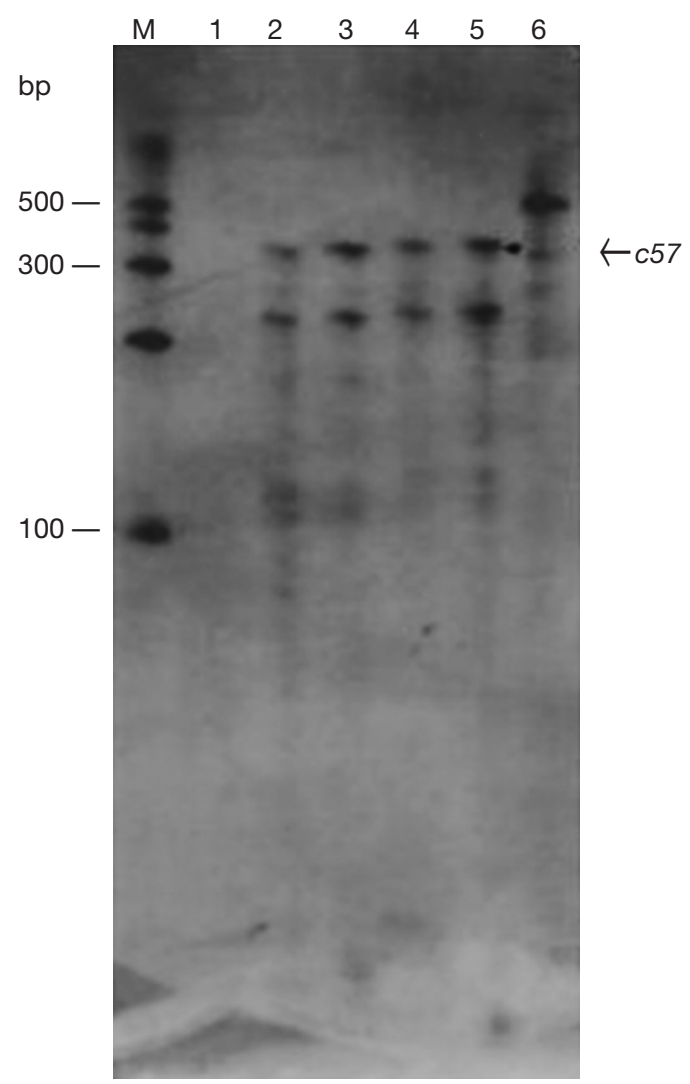

Fig. 3. Detection of $c 57$ mRNA in muscle and hepatopancreatic tissue of Metschnikowia bicuspidata-infected Macrobrachium rosenbergii prawns by ribonuclease protection assay (RPA). RNA from hepatopancreas of healthy prawns (lane 1), muscle and hepatopancreas of naturally infected prawns (lanes 2 and 3, respectively) and muscle and hepatopancreas of experimentally infected prawns (lanes 4 and 5, respectively) were hybridized with a digoxigenin (DIG)-labeled $464 \mathrm{nt}$ full-length c57 antisense riboprobe (lane 6). Protected c57 mRNA fragments were separated on a $6 \%$ polyacrylamide-urea gel, and visualized using a DIG Detection Kit (Roche Molecular Biochemicals). Lane M contains a $100 \mathrm{nt}$ size marker purchased from Ambion infection as a result of involvement in repair of tissues damaged by the yeast. The RPA data revealed another $230 \mathrm{bp}$ message (lanes 2 to 5 ) that was not identified by the DD. Lack of expression for this message in DD may be due to poor optimization of conditions for the separation of DNA within the size range of $230 \mathrm{bp}$ (i.e. DNA smearing seen on lanes 2 to 5 and 7 to 10, Fig. 1). As this $230 \mathrm{bp}$ fragment was expressed together with $c 57$ in the RPA, it is possible that both genes respond in a coordinated manner to $M$. bicuspidata infection. Further characterization of this DD fragment using different conditions would be required. RPA of hepatopancreatic tissue from healthy prawns failed to reveal any signal (lane 1). Taken together, our data demonstrate that ontogenic expression of $c 57$ in the muscle and hepatopancreas of giant freshwater prawns $M$. rosenbergii was a consequence of the $M$. bicuspidata infection.

Ubiquitous expression of actin has been found in mammals, Drosophila and crustaceans and the nucleotide sequences of their actin genes show high homology (Kang \& Naya 1993). In crustaceans, actin is believed to play an important role in developing cysts during the embryonic stage. Evidence has shown that at nauplius larva stage of the brine shrimp Artemia salina embryos, the proportion of actin mRNA and protein is increased 3-fold above levels found in the dormant cyst (Grosfeld \& Littauer 1976). It was suggested that at the nauplius stage, actin may participate with myosin and other proteins to form muscles and this correlates with the acquisition of motility during this free-swimming larval stage (Grosfeld \& Littauer 1976).

In addition to tissue repair, it is possible that expression of $c 57$ may have some functional role in protecting Macrobrachium rosenbergii against external agents like the yeast Metschnikowia bicuspidata. The issue might be examined by employing in situ hybridization analysis to identify the cellular location of $c 57$ expression. There is also evidence that expression of some immune-related proteins can be stimulated by yeast infections in shrimp. For example, Sequeira et al. (1996) reported that proliferating haemocytes were significantly increased in Marsupenaeus japonicus after stimulation by p43, an immunosuppressive lymphocyte mitogenic protein produced by Candida albicans. A similar response resulted from infection with Fusarium spp. (Sequeira et al. 1996). It is also known that betaglucan from yeast can initiate the shrimp prophenoloxidase (ProPO) defense cascade (Sritunyalucksana \& Soderhall 2000). However, no role for actin has yet been identified in the shrimp immune response.

Acknowledgements. This work was supported by National Science Council Grants NSC91-2313-B-020-028 and NSC922313-B-020-021, Taiwan, ROC. 


\section{LITERATURE CITED}

Altschul SF, Gish W, Miller W, Myers EW, Lipman DJ (1990) Basic local alignment search tool. J Mol Biol 215: 403-410

Bassam BJ, Caetano-Anolles G, Gresshoff PM (1991) Fast and sensitive silver staining of DNA in polyacrylamide gels. Anal Biochem 196:80-83

Chen SC, Chen TH, Wang PC, Chen YC, Huang JP, Lin YD, Chaung HC, Liaw LL (2003) Metschnikowia bicuspidata and Enterococcus faecium coinfection in giant freshwater prawn Macrobrachium rosenbergii. Dis Aquat Org 55: 161-167

Cheng W, Chen JC (1998) Isolation and characterization of an Enterococcus-like bacterium causing muscle necrosis and mortality in Macrobrachium rosenbergii in Taiwan. Dis Aquat Org 34:93-101

Doss RP (1996) Differential display without radioactivitya modified procedure. BioTechniques 21:408-410

Grosfeld H, Littauer UZ (1976) The translation in vitro of mRNA from developing cysts of Artemia salina. Eur J Biochem 70:589-599

Kang WK, Naya Y (1993) Sequence of the cDNA encoding an actin homolog in the crayfish Procambarus clarkii. Gene 133:303-304

Kurtzman CP, Robnett CJ (1997) Identification of clinically important ascomycetous yeasts based on nucleotide divergence in the $5^{\prime}$ end of the large-subunit (26S) ribosomal DNA gene. J Clin Microbiol 35:1216-1223

Liang P, Pardee AB (1992) Differential display of eukaryotic messenger RNA by means of polymerase chain reaction. Science 157:967-971

Liang P, Averboukh L, Pardee AB (1993) Distribution and cloning of eukaryotic mRNAs by means of differential

Editorial responsibility: Timothy Flegel,

Bangkok, Thailand display: refinements and optimization. Nucleic Acids Res 21:3269-3275

Lu CC, Tang FJ, Yoichiro U, Kou GH, Chen SN (1997) Yeast infection in prawns (Macrobrachium rosenbergii de Man) in Taiwan. Acta Zool Taiwanica 8:33-45

Lu CC, Tang KFG, Chen SN (1998) Identification and genetic characterization of yeast isolated from freshwater prawns, Macrobrachium rosenbergii de Man, in Taiwan. J Fish Dis 21:185-192

New MB (1995) Status of freshwater prawn farming: a review. Aquacult Res 3:46-65

Sequeira T, Tavares D, Arala-Chaves M (1996) Evidence for circulating hemocyte proliferation in the shrimp Penaeus japonicus. Dev Comp Immunol 20:97-104

Sritunyalucksana K, Soderhall K (2000) The proPO and clotting system in crustaceans. Aquaculture 191:53-69

Su HY, Palmer BR, Wellby PM, Bickerstaffe R, Hickford JGH (2001) Differential expression of a gene homologous to a G-protein gene in neonatal mouse skin during development of hair follicles. J Dermatol Sci 25:10-19

Su HY, Cheng WTK, Chen SC, Lin CT, Lien YY, Liu HJ, Gilmour RS (2004) Mouse keratinocytes express c98, a novel gene homologous to $b c l-2$ that is stimulated by insulin-like growth factor 1 and prevents dexamethasoneinduced apoptosis. Biochim Biophys Acta 1676:127-137

Tartof KD, Hobbs CA (1987) Improved media for growing plasmid and cosmid clones. Bethesda Res Lab Focus 9:12

Weaver KR, Caetano-Anolles G, Gresshoff PM, Gallahan LM (1994) Isolation and cloning of DNA amplification products from silver-stained polyacrylamide gels. BioTechniques 16:226-227

Wundrack I, Dooley S (1992) Nonradioactive ribunuclease protection analysis using digoxygenin labeling chemiluminescent detection. Electrophoresis 13:637-638

Submitted: August 17, 2004; Accepted: March 22, 2005

Proofs received from author(s): August 25, 2005 